\title{
Article
}

\section{The forgotten index of complement graph operations and its applications of molecular graph}

\author{
Mohammed Saad Alsharafi ${ }^{1, *}$, Mahioub Mohammed Shubatah ${ }^{2}$ and Abdu Qaid Alameri ${ }^{3}$ \\ 1 Department of Mathematics, Faculty of Education, Art and Science, University of Sheba Region, Yemen. \\ 2 Department of Studies in Mathematics, Faculty of Science and Education, AL-Baida University, AL-Baida-Yemen. \\ 3 Department of BME, Faculty of Engineering, University of Science and Technology, Yemen. \\ * Correspondence: alsharafi205010@gmail.com
}

Received: 6 August 2020; Accepted: 23 September 2020; Published: 24 October 2020.

\begin{abstract}
A topological index of graph $G$ is a numerical parameter related to graph which characterizes its molecular topology and is usually graph invariant. Topological indices are widely used to determine the correlation between the specific properties of molecules and the biological activity with their configuration in the study of quantitative structure-activity relationships (QSARs). In this paper some basic mathematical operations for the forgotten index of complement graph operations such as join $\overline{G_{1}+G_{2}}$, tensor product $\overline{G_{1} \otimes G_{2}}$, Cartesian product $\overline{G_{1} \times G_{2}}$, composition $\overline{G_{1} \circ G_{2}}$, strong product $\overline{G_{1} * G_{2}}$, disjunction $\overline{G_{1} \vee G_{2}}$ and symmetric difference $\overline{G_{1} \oplus G_{2}}$ will be explained. The results are applied to molecular graph of nanotorus and titania nanotubes.
\end{abstract}

Keywords: Forgotten index, Zagreb indices, complement graph, graph operation.

MSC: $05 C 92$.

\section{Introduction}

$\mathbf{T}$ heory of chemical graphs is the branch of mathematical chemistry that applies theory of graphs to mathematical modeling of chemical phenomena. In chemical graph theory a molecular graph is a simple graph in which the vertices and edges represent atoms and chemical bonds between them. In this paper, $G$ be a simple connected graph with vertex set $V(G)$ and edge set $E(G)$. The number of elements in $V(G)$ and $E(G)$ is represented as $|V(G)|$ and $|E(G)|$, respectively. For a vertex $u \in V(G)$, the number of vertices adjacent to the vertex $u$ is called the degree of $u$, denoted by $\delta_{G}(u)$. The complement of $G$, denoted by $\bar{G}$, is a simple graph on the same set of vertices $V(G)$ in which two vertices $u$ and $v$ are adjacent, i.e., connected by an edge $u v$, if and only if they are not adjacent in $G$. Hence, $u v \in E(\bar{G})$, if and only if $u v \notin E(G)$. Obviously $E(G) \cup E(\bar{G})=E\left(K_{n}\right)$, and $\bar{m}=|E(\bar{G})|=\left(\begin{array}{c}n \\ 2\end{array}\right)-m$, the degree of a vertex $u$ in $\bar{G}$, is the number of edges incident to $u$, denoted by $\delta_{\bar{G}}(u)=n-1-\delta_{G}(u)$ [1]. The well-known Zagreb indices introduced in [2] are among the most important topological indices. The first and second Zagreb indices $M_{1}$ and $M_{2}$, respectively, are defined for a molecular graph $\mathrm{G}$ as:

$$
M_{1}(G)=\sum_{v \in V(G)} \delta_{G}^{2}(v)=\sum_{u v \in E(G)}\left[\delta_{G}(u)+\delta_{G}(v)\right], \quad M_{2}(G)=\sum_{u v \in E(G)} \delta_{G}(u) \delta_{G}(v) .
$$

The first and second Zagreb coindices have been introduced by Ashrafi et al., in 2010 [3], they are respectively defined as:

$$
\bar{M}_{1}(G)=\sum_{u v \notin E(G)}\left[\delta_{G}(u)+\delta_{G}(v)\right], \quad \bar{M}_{2}(G)=\sum_{u v \notin E(G)} \delta_{G}(u) \delta_{G}(v) .
$$

Furtula and Gutman in 2015 introduced forgotten index (F-index) [4] which is defined as:

$$
F(G)=\sum_{v \in V(G)} \delta_{G}^{3}(v)=\sum_{u v \in E(G)}\left(\delta_{G}^{2}(u)+\delta_{G}^{2}(v)\right) .
$$


De et al., in 2016 defined forgotten coindex (F-coindex)[5] which is defined as:

$$
\bar{F}(G)=\sum_{u v \notin E(G)}\left(\delta_{G}^{2}(u)+\delta_{G}^{2}(v)\right) .
$$

Then, De et al., in 2016 [6] computed the forgotten index of join $G_{1}+G_{2}$, tensor product $G_{1} \otimes G_{2}$, Cartesian product $G_{1} \times G_{2}$, composition $G_{1} \circ G_{2}$, strong product $G_{1} * G_{2}$, disjunction $G_{1} \vee G_{2}$ and symmetric difference $G_{1} \oplus G_{2}$ of two graphs. Here we continue this line of research by exploring the behavior of the forgotten index under the same operations of complement graphs. The results are applied to molecular graph of nanotorus and titania nanotubes. In recent years, there has been considerable interest in general problems of determining topological indices and their operations [1,7-9].

\section{Preliminaries}

In this section we give some basic and preliminary concepts which we shall use later.

Lemma 1. [10,11] Let $G_{1}$ and $G_{2}$ be two connected graphs with $\left|V\left(G_{1}\right)\right|=n_{1},\left|V\left(G_{2}\right)\right|=n_{2},\left|E\left(G_{1}\right)\right|=m_{1}$, and $\left|E\left(G_{2}\right)\right|=m_{2}$. Then

1. $\left|V\left(G_{1} \times G_{2}\right)\right|=\left|V\left(G_{1} \vee G_{2}\right)\right|=\left|V\left(G_{1} \circ G_{2}\right)\right|=\left|V\left(G_{1} \otimes G_{2}\right)\right|=\left|V\left(G_{1} * G_{2}\right)\right|=\left|V\left(G_{1} \oplus G_{2}\right)\right|=n_{1} n_{2}$, $\left|V\left(G_{1}+G_{2}\right)\right|=n_{1}+n_{2}$,

2. $\left|E\left(G_{1} \times G_{2}\right)\right|=m_{1} n_{2}+n_{1} m_{2}$ ，

$\left|E\left(G_{1} * G_{2}\right)\right|=m_{1} n_{2}+n_{1} m_{2}+2 m_{1} m_{2}$

$\left|E\left(G_{1}+G_{2}\right)\right|=m_{1}+m_{2}+n_{1} n_{2}$,

$\left|E\left(G_{1} \circ G_{2}\right)\right|=m_{1} n_{2}^{2}+m_{2} n_{1}$

$\left|E\left(G_{1} \vee G_{2}\right)\right|=m_{1} n_{2}^{2}+m_{2} n_{1}^{2}-2 m_{1} m_{2}$,

$\left|E\left(G_{1} \otimes G_{2}\right)\right|=2 m_{1} m_{2}$,

$\left|E\left(G_{1} \oplus G_{2}\right)\right|=m_{1} n_{2}^{2}+m_{2} n_{1}^{2}-4 m_{1} m_{2}$.

Proposition 1. [12] Let $G$ be a simple graph on $n$ vertices and $m$ edges. Then.

$$
F(\bar{G})=n(n-1)^{3}-6 m(n-1)^{2}+3(n-1) M_{1}(G)-F(G) .
$$

\section{Discussion and main results}

In this section, we study the forgotten index of various complement graph binary operations such as join, tensor product, Cartesian product, composition, strong product, disjunction and symmetric difference of two simple connected graphs. We use the notation $V\left(G_{i}\right)$ for the vertex set, $E\left(G_{i}\right), E\left(\bar{G}_{i}\right)$ for the edge sets, $n_{i}$ for the number of vertices and $m_{i}, \bar{m}_{i}$ for the number of edges of the graph $G_{i}, \bar{G}_{i}$ respectively. All graphs here offer are simple graphs.

Definition 1 (Join). The join $G_{1}+G_{2}$ of two graphs $G_{1}$ and $G_{2}$ is a graph with vertex set $V\left(G_{1}+G_{2}\right)=$ $V\left(G_{1}\right) \cup V\left(G_{2}\right)$ and edge set $E\left(G_{1}\right) \cup E\left(G_{1}\right) \cup\left\{u v \mid u \in V\left(G_{1}\right), v \in V\left(G_{2}\right)\right\}$.

Theorem 1. The $F-$ index of the complement of $G_{1}+G_{2}$ is given by

$$
\begin{aligned}
F\left(\overline{G_{1}+G_{2}}\right)= & \left(n_{1}+n_{2}\right)\left(n_{1}+n_{2}-1\right)^{3}-6\left(m_{1}+m_{2}+n_{1} n_{2}\right)\left(n_{1}+n_{2}-1\right)^{2} \\
& +3\left(n_{1}+n_{2}-1\right)\left[M_{1}\left(G_{1}\right)+M_{1}\left(G_{2}\right)+n_{1} n_{2}^{2}+n_{2} n_{1}^{2}+4 m_{1} n_{2}+4 m_{2} n_{1}\right] \\
& -\left[F\left(G_{1}\right)+F\left(G_{2}\right)+3 n_{2} M_{1}\left(G_{1}\right)+3 n_{1} M_{1}\left(G_{2}\right)+6 n_{2}^{2} m_{1}+6 n_{1}^{2} m_{2}+n_{1} n_{2}^{3}+n_{2} n_{1}^{3}\right] .
\end{aligned}
$$

Proof. From Proposition 1, we have $F(\bar{G})=n(n-1)^{3}-6 m(n-1)^{2}+3(n-1) M_{1}(G)-F(G)$, and since $M_{1}\left(G_{1}+G_{2}\right)=M_{1}\left(G_{1}\right)+M_{1}\left(G_{2}\right)+n_{1} n_{2}^{2}+n_{2} n_{1}^{2}+4 m_{1} n_{2}+4 m_{2} n_{1}$, given in [10], $F\left(G_{1}+G_{2}\right)=F\left(G_{1}\right)+$ 
$F\left(G_{2}\right)+3 n_{2} M_{1}\left(G_{1}\right)+3 n_{1} M_{1}\left(G_{2}\right)+6 n_{2}^{2} m_{1}+6 n_{1}^{2} m_{2}+n_{1} n_{2}^{3}+n_{2} n_{1}^{3}$, given in [6]. and $\left|E\left(G_{1}+G_{2}\right)\right|=m_{1}+$ $m_{2}+n_{1} n_{2}, \quad\left|V\left(G_{1}+G_{2}\right)\right|=n_{1}+n_{2}$ given in Lemma 1. Then

$$
\begin{aligned}
F\left(\overline{G_{1}+G_{2}}\right)= & \left|V\left(G_{1}+G_{2}\right)\right|\left(\left|V\left(G_{1}+G_{2}\right)\right|-1\right)^{3}-6\left|E\left(G_{1}+G_{2}\right)\right|\left(\left|V\left(G_{1}+G_{2}\right)\right|-1\right)^{2} \\
& +3\left(\left|V\left(G_{1}+G_{2}\right)\right|-1\right) M_{1}\left(G_{1}+G_{2}\right)-F\left(G_{1}+G_{2}\right) \\
= & \left(n_{1}+n_{2}\right)\left(n_{1}+n_{2}-1\right)^{3}-6\left(m_{1}+m_{2}+n_{1} n_{2}\right)\left(n_{1}+n_{2}-1\right)^{2} \\
& +3\left(n_{1}+n_{2}-1\right)\left[M_{1}\left(G_{1}\right)+M_{1}\left(G_{2}\right)+n_{1} n_{2}^{2}+n_{2} n_{1}^{2}+4 m_{1} n_{2}+4 m_{2} n_{1}\right] \\
& -\left[F\left(G_{1}\right)+F\left(G_{2}\right)+3 n_{2} M_{1}\left(G_{1}\right)+3 n_{1} M_{1}\left(G_{2}\right)+6 n_{2}^{2} m_{1}+6 n_{1}^{2} m_{2}+n_{1} n_{2}^{3}+n_{2} n_{1}^{3}\right] .
\end{aligned}
$$

Definition 2 (Tensor product). The tensor product $G_{1} \otimes G_{2}$ of two graphs $G_{1}$ and $G_{2}$ is the graph with vertex set $V\left(G_{1}\right) \times V\left(G_{2}\right)$ and any two vertices $\left(u_{1}, v_{1}\right)$ and $\left(u_{2}, v_{2}\right)$ are adjacent if and only if $u_{1} u_{2} \in E\left(G_{1}\right)$ and $v_{1} v_{2} \in E\left(G_{2}\right)$.

Theorem 2. The $F-$ index of the complement of $G_{1} \otimes G_{2}$ is given by

$$
F\left(\overline{G_{1} \otimes G_{2}}\right)=n_{1} n_{2}\left(n_{1} n_{2}-1\right)^{3}-12 m_{1} m_{2}\left(n_{1} n_{2}-1\right)^{2}+3\left(n_{1} n_{2}-1\right) M_{1}\left(G_{1}\right) M_{1}\left(G_{2}\right)-F\left(G_{1}\right) F\left(G_{2}\right) .
$$

Proof. From Proposition 1 we have $F(\bar{G})=n(n-1)^{3}-6 m(n-1)^{2}+3(n-1) M_{1}(G)-F(G)$, and since $M_{1}\left(G_{1} \otimes G_{2}\right)=M_{1}\left(G_{1}\right) M_{1}\left(G_{2}\right)$ given in [13], $F\left(G_{1} \otimes G_{2}\right)=F\left(G_{1}\right) F\left(G_{2}\right)$ given in [6] and $\left|E\left(G_{1} \otimes G_{2}\right)\right|=$ $2 m_{1} m_{2}, \quad\left|V\left(G_{1} \otimes G_{2}\right)\right|=n_{1} n_{2}$ given in Lemma 1. Then

$$
\begin{aligned}
F\left(\overline{G_{1} \otimes G_{2}}\right)= & \left|V\left(G_{1} \otimes G_{2}\right)\right|\left(\left|V\left(G_{1} \otimes G_{2}\right)\right|-1\right)^{3}-6\left|E\left(G_{1} \otimes G_{2}\right)\right|\left(\left|V\left(G_{1} \otimes G_{2}\right)\right|-1\right)^{2} \\
& +3\left(\left|V\left(G_{1} \otimes G_{2}\right)\right|-1\right) M_{1}\left(G_{1} \otimes G_{2}\right)-F\left(G_{1} \otimes G_{2}\right) \\
= & n_{1} n_{2}\left(n_{1} n_{2}-1\right)^{3}-12 m_{1} m_{2}\left(n_{1} n_{2}-1\right)^{2}+3\left(n_{1} n_{2}-1\right) M_{1}\left(G_{1}\right) M_{1}\left(G_{2}\right)-F\left(G_{1}\right) F\left(G_{2}\right) .
\end{aligned}
$$

Definition 3 (Cartesian product). The Cartesian product $G_{1} \times G_{2}$, of two simple and connected graphs $G_{1}$ and $G_{2}$ has the vertex $\operatorname{set} V\left(G_{1} \times G_{2}\right)=V\left(G_{1}\right) \times V\left(G_{2}\right)$ and $(a, x)(b, y)$ is an edge of $G_{1} \times G_{2}$ if $a=b$ and $x y \in E\left(G_{2}\right)$, or $a b \in E\left(G_{1}\right)$ and $x=y$.

Theorem 3. The $F-$ index of the complement of $G_{1} \times G_{2}$ is given by

$$
\begin{aligned}
F\left(\overline{G_{1} \times G_{2}}\right)= & n_{1} n_{2}\left(n_{1} n_{2}-1\right)^{3}-6\left(m_{1} n_{2}+n_{1} m_{2}\right)\left(n_{1} n_{2}-1\right)^{2}+3\left(n_{1} n_{2}-1\right)\left[n_{2} M_{1}\left(G_{1}\right)+n_{1} M_{1}\left(G_{2}\right)+8 m_{1} m_{2}\right] \\
& -\left[n_{2} F\left(G_{1}\right)+n_{1} F\left(G_{2}\right)+6 m_{2} M_{1}\left(G_{1}\right)+6 m_{1} M_{1}\left(G_{2}\right)\right] .
\end{aligned}
$$

Proof. From Proposition 1, we have $F(\bar{G})=n(n-1)^{3}-6 m(n-1)^{2}+3(n-1) M_{1}(G)-F(G)$, and since $M_{1}\left(G_{1} \times G_{2}\right)=n_{2} M_{1}\left(G_{1}\right)+n_{1} M_{1}\left(G_{2}\right)+8 m_{1} m_{2}$, given in [14], $F\left(G_{1} \times G_{2}\right)=n_{2} F\left(G_{1}\right)+n_{1} F\left(G_{2}\right)+$ $6 m_{2} M_{1}\left(G_{1}\right)+6 m_{1} M_{1}\left(G_{2}\right)$, given in [6]. and $\left|E\left(G_{1} \times G_{2}\right)\right|=m_{1} n_{2}+n_{1} m_{2}, \quad\left|V\left(G_{1} \times G_{2}\right)\right|=n_{1} n_{2}$ given in Lemma 1. Then

$$
\begin{aligned}
F\left(\overline{G_{1} \times G_{2}}\right)= & \left|V\left(G_{1} * G_{2}\right)\right|\left(\left|V\left(G_{1} * G_{2}\right)\right|-1\right)^{3}-6\left|E\left(G_{1} * G_{2}\right)\right|\left(\left|V\left(G_{1} * G_{2}\right)\right|-1\right)^{2} \\
& +3\left(\left|V\left(G_{1} * G_{2}\right)\right|-1\right) M_{1}\left(G_{1} * G_{2}\right)-F\left(G_{1} * G_{2}\right) \\
= & n_{1} n_{2}\left(n_{1} n_{2}-1\right)^{3}-6\left(m_{1} n_{2}+n_{1} m_{2}\right)\left(n_{1} n_{2}-1\right)^{2} \\
& +3\left(n_{1} n_{2}-1\right)\left[n_{2} M_{1}\left(G_{1}\right)+n_{1} M_{1}\left(G_{2}\right)+8 m_{1} m_{2}\right] \\
& -\left[n_{2} F\left(G_{1}\right)+n_{1} F\left(G_{2}\right)+6 m_{2} M_{1}\left(G_{1}\right)+6 m_{1} M_{1}\left(G_{2}\right)\right] .
\end{aligned}
$$


Definition 4 (Composition). The composition $G_{1} \circ G_{2}$, of two simple and connected graphs $G_{1}$ and $G_{2}$ with disjoint vertex sets $V\left(G_{1}\right)$ and $V\left(G_{2}\right)$ and edge sets $E\left(G_{1}\right)$ and $E\left(G_{2}\right)$ is the graph with vertex set $V\left(G_{1}\right) \times$ $V\left(G_{2}\right)$ and $u=\left(u_{1}, v_{1}\right)$ is adjacent with $v=\left(u_{2}, v_{2}\right)$ whenever $\left(u_{1}\right.$ is adjacent with $\left.u_{2}\right)$ or $\left\{u_{1}=u_{2}\right.$ and $v_{1}$ is adjacent with $\left.v_{2}\right\}$.

Theorem 4. The $F$ - index of the complement of $G_{1} \circ G_{2}$ is given by

$$
\begin{aligned}
F\left(\overline{G_{1} \circ G_{2}}\right)= & n_{1} n_{2}\left(n_{1} n_{2}-1\right)^{3}-6\left(m_{1} n_{2}^{2}+m_{2} n_{1}\right)\left(n_{1} n_{2}-1\right)^{2} \\
& +3\left(n_{1} n_{2}-1\right)\left[n_{2}^{3} M_{1}\left(G_{1}\right)+n_{1} M_{1}\left(G_{2}\right)+8 n_{2} m_{2} m_{1}\right] \\
& -\left[n_{2}^{4} F\left(G_{1}\right)+n_{1} F\left(G_{2}\right)+6 n_{2}^{2} m_{2} M_{1}\left(G_{1}\right)+6 n_{2} m_{1} M_{1}\left(G_{2}\right)\right] .
\end{aligned}
$$

Proof. From Proposition 1, we have $F(\bar{G})=n(n-1)^{3}-6 m(n-1)^{2}+3(n-1) M_{1}(G)-F(G)$, and since $M_{1}\left(G_{1} \circ G_{2}\right)=n_{2}^{3} M_{1}\left(G_{1}\right)+n_{1} M_{1}\left(G_{2}\right)+8 n_{2} m_{2} m_{1}$, given in [14]. $F\left(G_{1} \circ G_{2}\right)=n_{2}^{4} F\left(G_{1}\right)+n_{1} F\left(G_{2}\right)+$ $6 n_{2}^{2} m_{2} M_{1}\left(G_{1}\right)+6 n_{2} m_{1} M_{1}\left(G_{2}\right)$, given in [6]. and $\left|E\left(G_{1} \circ G_{2}\right)\right|=m_{1} n_{2}^{2}+m_{2} n_{1}, \quad\left|V\left(G_{1} \circ G_{2}\right)\right|=n_{1} n_{2}$ given in Lemma 1. Then

$$
\begin{aligned}
F\left(\overline{G_{1} \circ G_{2}}\right)= & \left|V\left(G_{1} \circ G_{2}\right)\right|\left(\left|V\left(G_{1} \circ G_{2}\right)\right|-1\right)^{3}-6\left|E\left(G_{1} \circ G_{2}\right)\right|\left(\left|V\left(G_{1} \circ G_{2}\right)\right|-1\right)^{2} \\
& +3\left(\left|V\left(G_{1} \circ G_{2}\right)\right|-1\right) M_{1}\left(G_{1} \circ G_{2}\right)-F\left(G_{1} \circ G_{2}\right) \\
= & n_{1} n_{2}\left(n_{1} n_{2}-1\right)^{3}-6\left(m_{1} n_{2}^{2}+m_{2} n_{1}\right)\left(n_{1} n_{2}-1\right)^{2} \\
& +3\left(n_{1} n_{2}-1\right)\left[n_{2}^{3} M_{1}\left(G_{1}\right)+n_{1} M_{1}\left(G_{2}\right)+8 n_{2} m_{2} m_{1}\right] \\
& -\left[n_{2}^{4} F\left(G_{1}\right)+n_{1} F\left(G_{2}\right)+6 n_{2}^{2} m_{2} M_{1}\left(G_{1}\right)+6 n_{2} m_{1} M_{1}\left(G_{2}\right)\right] .
\end{aligned}
$$

Definition 5 (Strong product). The strong product $G_{1} * G_{2}$, of two simple and connected graphs $G_{1}$ and $G_{2}$ is a graph with vertex set $V\left(G_{1} * G_{2}\right)=V\left(G_{1}\right) \times V\left(G_{2}\right)$ and any two vertices $\left(u_{1}, v_{1}\right)$ and $\left(u_{2}, v_{2}\right)$ are adjacent if and only if $\left\{u_{1}=u_{2} \in V\left(G_{1}\right)\right.$ and $\left.v_{1} v_{2} \in E\left(G_{2}\right)\right\}$ or $\left\{v_{1}=v_{2} \in V\left(G_{2}\right)\right.$ and $\left.u_{1} u_{2} \in E\left(G_{1}\right)\right\}$.

Proposition 2. [15] Let $G_{1}, G_{2}$ be two graphs with $n_{1}, n_{2}$ vertices and $m_{1}, m_{2}$ edges, respectively. Then

$$
M_{1}\left(G_{1} * G_{2}\right)=\left(n_{2}+6 m_{2}\right) M_{1}\left(G_{1}\right)+8 m_{2} m_{1}+\left(6 m_{1}+n_{1}\right) M_{1}\left(G_{2}\right)+2 M_{1}\left(G_{1}\right) M_{1}\left(G_{2}\right) .
$$

Theorem 5. The $F$ - index of the complement of $G_{1} * G_{2}$ is given by

$$
\begin{aligned}
F\left(\overline{G_{1} * G_{2}}\right)= & n_{1} n_{2}\left(n_{1} n_{2}-1\right)^{3}-6\left(m_{1} n_{2}+n_{1} m_{2}+2 m_{1} m_{2}\right)\left(n_{1} n_{2}-1\right)^{2} \\
& +3\left(n_{1} n_{2}-1\right)\left[\left(n_{2}+6 m_{2}\right) M_{1}\left(G_{1}\right)+8 m_{2} m_{1}+\left(6 m_{1}+n_{1}\right) M_{1}\left(G_{2}\right)+2 M_{1}\left(G_{1}\right) M_{1}\left(G_{2}\right)\right] \\
& -\left[n_{2} F\left(G_{1}\right)+n_{1} F\left(G_{2}\right)+F\left(G_{1}\right) F\left(G_{2}\right)+6 m_{2} M_{1}\left(G_{1}\right)+6 m_{1} M_{1}\left(G_{2}\right)\right. \\
& \left.+6 m_{2} F\left(G_{1}\right)+6 m_{1} F\left(G_{2}\right)+3 F\left(G_{2}\right) M_{1}\left(G_{1}\right)+3 F\left(G_{1}\right) M_{1}\left(G_{2}\right)+6 M_{1}\left(G_{1}\right) M_{1}\left(G_{2}\right)\right] .
\end{aligned}
$$

Proof. From Proposition 1, we have $F(\bar{G})=n(n-1)^{3}-6 m(n-1)^{2}+3(n-1) M_{1}(G)-F(G)$, and since $M_{1}\left(G_{1} * G_{2}\right)=\left(n_{2}+6 m_{2}\right) M_{1}\left(G_{1}\right)+8 m_{2} m_{1}+\left(6 m_{1}+n_{1}\right) M_{1}\left(G_{2}\right)+2 M_{1}\left(G_{1}\right) M_{1}\left(G_{2}\right)$, given in Proposition 2, and by [6] we have

$$
\begin{aligned}
F\left(G_{1} * G_{2}\right)= & n_{2} F\left(G_{1}\right)+n_{1} F\left(G_{2}\right)+F\left(G_{1}\right) F\left(G_{2}\right)+6 m_{2} M_{1}\left(G_{1}\right)+6 M_{1}\left(G_{1}\right) M_{1}\left(G_{2}\right) \\
& +6 m_{1} M_{1}\left(G_{2}\right)+6 m_{2} F\left(G_{1}\right)+6 m_{1} F\left(G_{2}\right)+3 F\left(G_{2}\right) M_{1}\left(G_{1}\right)+3 F\left(G_{1}\right) M_{1}\left(G_{2}\right) .
\end{aligned}
$$

And since $\left|E\left(G_{1} * G_{2}\right)\right|=m_{1} n_{2}+n_{1} m_{2}+2 m_{1} m_{2}, \quad\left|V\left(G_{1} * G_{2}\right)\right|=n_{1} n_{2}$, given in Lemma 1 . Then

$$
\begin{aligned}
F\left(\overline{G_{1} * G_{2}}\right)= & \left|V\left(G_{1} * G_{2}\right)\right|\left(\left|V\left(G_{1} * G_{2}\right)\right|-1\right)^{3}-6\left|E\left(G_{1} * G_{2}\right)\right|\left(\left|V\left(G_{1} * G_{2}\right)\right|-1\right)^{2} \\
& +3\left(\left|V\left(G_{1} * G_{2}\right)\right|-1\right) M_{1}\left(G_{1} * G_{2}\right)-F\left(G_{1} * G_{2}\right)
\end{aligned}
$$




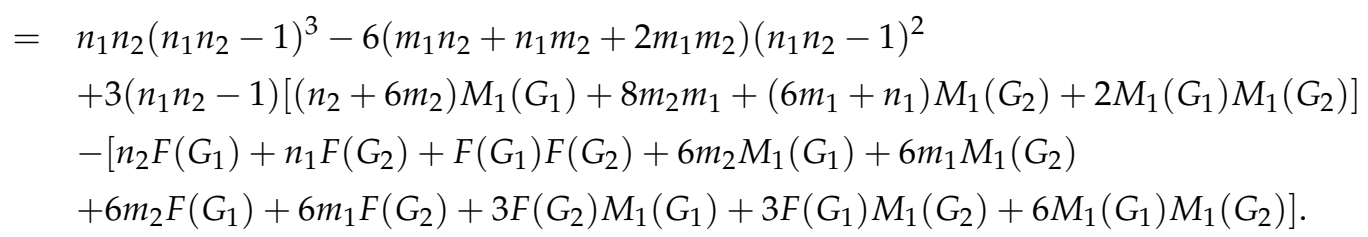

Definition 6 (Disjunction). The disjunction $G_{1} \vee G_{2}$ of graphs $G_{1}$ and $G_{2}$ is the graph with vertex set $V\left(G_{1}\right) \times$ $V\left(G_{2}\right)$ and $\left(u_{1}, v_{1}\right)$ is adjacent with $\left(u_{2}, v_{2}\right)$, whenever $\left(u_{1}, u_{2}\right) \in E\left(G_{1}\right)$ or $\left(v_{1}, v_{2}\right) \in E\left(G_{2}\right)$.

Theorem 6. The first Zagreb index of $G_{1} \vee G_{2}$ is given by

$$
M_{1}\left(G_{1} \vee G_{2}\right)=\left[n_{2}^{3}-4 n_{2} m_{2}\right] M_{1}\left(G_{1}\right)+\left[n_{1}^{3}-4 n_{1} m_{1}\right] M_{1}\left(G_{2}\right)+8 n_{1} n_{2} m_{1} m_{2}+M_{1}\left(G_{1}\right) M_{1}\left(G_{2}\right) .
$$

Theorem 7. The $F$ - index of the complement of $G_{1} \vee G_{2}$ is given by

$$
\begin{aligned}
F\left(\overline{G_{1} \vee G_{2}}\right)= & n_{1} n_{2}\left(n_{1} n_{2}-1\right)^{3}-6\left[m_{1} n_{2}^{2}+m_{2} n_{1}^{2}-2 m_{1} m_{2}\right]\left(n_{1} n_{2}-1\right)^{2} \\
& +3\left(n_{1} n_{2}-1\right)\left[\left[n_{2}^{3}-4 n_{2} m_{2}\right] M_{1}\left(G_{1}\right)+\left[n_{1}^{3}-4 n_{1} m_{1}\right] M_{1}\left(G_{2}\right)\right. \\
& \left.+8 n_{1} n_{2} m_{1} m_{2}+M_{1}\left(G_{1}\right) M_{1}\left(G_{2}\right)\right]-\left[n_{2}^{4} F\left(G_{1}\right)+n_{1}^{4} F\left(G_{2}\right)-F\left(G_{1}\right) F\left(G_{2}\right)\right. \\
& +6 n_{1} n_{2}^{2} m_{2} M_{1}\left(G_{1}\right)+6 n_{2} n_{1}^{2} m_{1} M_{1}\left(G_{2}\right)+3 n_{2} F\left(G_{1}\right) M_{1}\left(G_{2}\right)+3 n_{1} F\left(G_{2}\right) M_{1}\left(G_{1}\right) \\
& \left.-6 n_{2}^{2} m_{2} F\left(G_{1}\right)-6 n_{1}^{2} m_{1} F\left(G_{2}\right)-6 n_{1} n_{2} M_{1}\left(G_{1}\right) M_{1}\left(G_{2}\right)\right] .
\end{aligned}
$$

Proof. From Proposition 1, we have $F(\bar{G})=n(n-1)^{3}-6 m(n-1)^{2}+3(n-1) M_{1}(G)-F(G)$, and by Theorem 6 and [6], respectively, we have

$$
\begin{aligned}
M_{1}\left(G_{1} \vee G_{2}\right)=\left[n_{2}^{3}-4 n_{2} m_{2}\right] M_{1}\left(G_{1}\right)+\left[n_{1}^{3}-4 n_{1} m_{1}\right] M_{1}\left(G_{2}\right)+8 n_{1} n_{2} m_{1} m_{2}+M_{1}\left(G_{1}\right) M_{1}\left(G_{2}\right) . \\
\begin{aligned}
F\left(G_{1} \vee G_{2}\right)= & n_{2}^{4} F\left(G_{1}\right)+n_{1}^{4} F\left(G_{2}\right)-F\left(G_{1}\right) F\left(G_{2}\right)+6 n_{1} n_{2}^{2} m_{2} M_{1}\left(G_{1}\right) \\
& +6 n_{2} n_{1}^{2} m_{1} M_{1}\left(G_{2}\right)+3 n_{2} F\left(G_{1}\right) M_{1}\left(G_{2}\right)+3 n_{1} F\left(G_{2}\right) M_{1}\left(G_{1}\right) \\
& -6 n_{2}^{2} m_{2} F\left(G_{1}\right)-6 n_{1}^{2} m_{1} F\left(G_{2}\right)-6 n_{1} n_{2} M_{1}\left(G_{1}\right) M_{1}\left(G_{2}\right) .
\end{aligned}
\end{aligned}
$$

And since $\left|E\left(G_{1} \vee G_{2}\right)\right|=m_{1} n_{2}^{2}+m_{2} n_{1}^{2}-2 m_{1} m_{2}, \quad\left|V\left(G_{1} \vee G_{2}\right)\right|=n_{1} n_{2}$ given in Lemma 1. Then

$$
\begin{aligned}
F\left(\overline{G_{1} \vee G_{2}}\right)= & \left|V\left(G_{1} \vee G_{2}\right)\right|\left(\left|V\left(G_{1} \vee G_{2}\right)\right|-1\right)^{3}-6\left|E\left(G_{1} \vee G_{2}\right)\right|\left(\left|V\left(G_{1} \vee G_{2}\right)\right|-1\right)^{2} \\
& +3\left(\left|V\left(G_{1} \vee G_{2}\right)\right|-1\right) M_{1}\left(G_{1} \vee G_{2}\right)-F\left(G_{1} \vee G_{2}\right) \\
= & n_{1} n_{2}\left(n_{1} n_{2}-1\right)^{3}-6\left[m_{1} n_{2}^{2}+m_{2} n_{1}^{2}-2 m_{1} m_{2}\right]\left(n_{1} n_{2}-1\right)^{2} \\
& +3\left(n_{1} n_{2}-1\right)\left[\left[n_{2}^{3}-4 n_{2} m_{2}\right] M_{1}\left(G_{1}\right)+\left[n_{1}^{3}-4 n_{1} m_{1}\right] M_{1}\left(G_{2}\right)\right. \\
& \left.+8 n_{1} n_{2} m_{1} m_{2}+M_{1}\left(G_{1}\right) M_{1}\left(G_{2}\right)\right]-\left[n_{2}^{4} F\left(G_{1}\right)+n_{1}^{4} F\left(G_{2}\right)-F\left(G_{1}\right) F\left(G_{2}\right)\right. \\
& +6 n_{1} n_{2}^{2} m_{2} M_{1}\left(G_{1}\right)+6 n_{2} n_{1}^{2} m_{1} M_{1}\left(G_{2}\right)+3 n_{2} F\left(G_{1}\right) M_{1}\left(G_{2}\right)+3 n_{1} F\left(G_{2}\right) M_{1}\left(G_{1}\right) \\
& \left.-6 n_{2}^{2} m_{2} F\left(G_{1}\right)-6 n_{1}^{2} m_{1} F\left(G_{2}\right)-6 n_{1} n_{2} M_{1}\left(G_{1}\right) M_{1}\left(G_{2}\right)\right] .
\end{aligned}
$$

Definition 7 (Symmetric difference). The symmetric difference $G_{1} \oplus G_{2}$, of two simple and connected graphs $G_{1}$ and $G_{2}$ is the graph with vertex set $V\left(G_{1}\right) \times V\left(G_{2}\right)$ and $E\left(G_{1} \oplus G_{2}\right)=\left(u_{1}, u_{2}\right)\left(v_{1}, v_{2}\right) \mid u_{1} v_{1} \in E\left(G_{1}\right)$ or $u_{2} v_{2} \in E\left(G_{2}\right)$ but not both.

Theorem 8. The first Zagreb index of $G_{1} \oplus G_{2}$ is given by

$$
M_{1}\left(G_{1} \oplus G_{2}\right)=\left[n_{2}^{3}-8 n_{2} m_{2}\right] M_{1}\left(G_{1}\right)+\left[n_{1}^{3}-8 n_{1} m_{1}\right] M_{1}\left(G_{2}\right)+8 n_{1} n_{2} m_{1} m_{2}+4 M_{1}\left(G_{1}\right) M_{1}\left(G_{2}\right) .
$$


Theorem 9. The $F-$ index of the complement of $G_{1} \oplus G_{2}$ is given by

$$
\begin{aligned}
F\left(\overline{G_{1} \oplus G_{2}}\right)= & n_{1} n_{2}\left(n_{1} n_{2}-1\right)^{3}-6\left[m_{1} n_{2}^{2}+m_{2} n_{1}^{2}-4 m_{1} m_{2}\right]\left(n_{1} n_{2}-1\right)^{2} \\
& +3\left(n_{1} n_{2}-1\right)\left[\left[n_{2}^{3}-8 n_{2} m_{2}\right] M_{1}\left(G_{1}\right)+\left[n_{1}^{3}-8 n_{1} m_{1}\right] M_{1}\left(G_{2}\right)\right. \\
& \left.+8 n_{1} n_{2} m_{1} m_{2}+4 M_{1}\left(G_{1}\right) M_{1}\left(G_{2}\right)\right]-\left[n_{2}^{4} F\left(G_{1}\right)+n_{1}^{4} F\left(G_{2}\right)\right. \\
& -8 F\left(G_{1}\right) F\left(G_{2}\right)+6 n_{1} n_{2}^{2} m_{2} M_{1}\left(G_{1}\right)+6 n_{2} n_{1}^{2} m_{1} M_{1}\left(G_{2}\right) \\
& +12 n_{2} F\left(G_{1}\right) M_{1}\left(G_{2}\right)+12 n_{1} F\left(G_{2}\right) M_{1}\left(G_{1}\right)-12 n_{2}^{2} m_{2} F\left(G_{1}\right) \\
& \left.-12 n_{1}^{2} m_{1} F\left(G_{2}\right)-12 n_{1} n_{2} M_{1}\left(G_{1}\right) M_{1}\left(G_{2}\right)\right]
\end{aligned}
$$

Proof. From Proposition 1, we have $F(\bar{G})=n(n-1)^{3}-6 m(n-1)^{2}+3(n-1) M_{1}(G)-F(G)$, and by Theorem 8 and [6], respectively, we have

$$
\begin{aligned}
M_{1}\left(G_{1} \oplus G_{2}\right)=\left[n_{2}^{3}-8 n_{2} m_{2}\right] M_{1}\left(G_{1}\right)+\left[n_{1}^{3}-8 n_{1} m_{1}\right] M_{1}\left(G_{2}\right)+8 n_{1} n_{2} m_{1} m_{2}+4 M_{1}\left(G_{1}\right) M_{1}\left(G_{2}\right) \\
\begin{aligned}
F\left(G_{1} \oplus G_{2}\right)= & n_{2}^{4} F\left(G_{1}\right)+n_{1}^{4} F\left(G_{2}\right)-8 F\left(G_{1}\right) F\left(G_{2}\right)+6 n_{1} n_{2}^{2} m_{2} M_{1}\left(G_{1}\right) \\
& +6 n_{2} n_{1}^{2} m_{1} M_{1}\left(G_{2}\right)+12 n_{2} F\left(G_{1}\right) M_{1}\left(G_{2}\right)+12 n_{1} F\left(G_{2}\right) M_{1}\left(G_{1}\right) \\
& -12 n_{2}^{2} m_{2} F\left(G_{1}\right)-12 n_{1}^{2} m_{1} F\left(G_{2}\right)-12 n_{1} n_{2} M_{1}\left(G_{1}\right) M_{1}\left(G_{2}\right)
\end{aligned}
\end{aligned}
$$

And since $\left|E\left(G_{1} \oplus G_{2}\right)\right|=m_{1} n_{2}^{2}+m_{2} n_{1}^{2}-4 m_{1} m_{2}, \quad\left|V\left(G_{1} \oplus G_{2}\right)\right|=n_{1} n_{2}$ given in Lemma 1. Then

$$
\begin{aligned}
F\left(\overline{G_{1} \oplus G_{2}}\right)= & \left|V\left(G_{1} \oplus G_{2}\right)\right|\left(\left|V\left(G_{1} \oplus G_{2}\right)\right|-1\right)^{3}-6\left|E\left(G_{1} \oplus G_{2}\right)\right|\left(\left|V\left(G_{1} \oplus G_{2}\right)\right|-1\right)^{2} \\
& +3\left(\left|V\left(G_{1} \oplus G_{2}\right)\right|-1\right) M_{1}\left(G_{1} \oplus G_{2}\right)-F\left(G_{1} \oplus G_{2}\right) \\
= & n_{1} n_{2}\left(n_{1} n_{2}-1\right)^{3}-6\left[m_{1} n_{2}^{2}+m_{2} n_{1}^{2}-4 m_{1} m_{2}\right]\left(n_{1} n_{2}-1\right)^{2} \\
& +3\left(n_{1} n_{2}-1\right)\left[\left[n_{2}^{3}-8 n_{2} m_{2}\right] M_{1}\left(G_{1}\right)+\left[n_{1}^{3}-8 n_{1} m_{1}\right] M_{1}\left(G_{2}\right)\right. \\
& \left.+8 n_{1} n_{2} m_{1} m_{2}+4 M_{1}\left(G_{1}\right) M_{1}\left(G_{2}\right)\right]-\left[n_{2}^{4} F\left(G_{1}\right)+n_{1}^{4} F\left(G_{2}\right)\right. \\
& -8 F\left(G_{1}\right) F\left(G_{2}\right)+6 n_{1} n_{2}^{2} m_{2} M_{1}\left(G_{1}\right)+6 n_{2} n_{1}^{2} m_{1} M_{1}\left(G_{2}\right) \\
& +12 n_{2} F\left(G_{1}\right) M_{1}\left(G_{2}\right)+12 n_{1} F\left(G_{2}\right) M_{1}\left(G_{1}\right)-12 n_{2}^{2} m_{2} F\left(G_{1}\right) \\
& \left.-12 n_{1}^{2} m_{1} F\left(G_{2}\right)-12 n_{1} n_{2} M_{1}\left(G_{1}\right) M_{1}\left(G_{2}\right)\right] .
\end{aligned}
$$

\section{Application}

In this section, the forgotten index have been investigated for complement titania $\mathrm{TiO}_{2}$ nanotubes and molecular graph of nanotorus.

Corollary 1. The forgotten index of complement $\mathrm{TiO}_{2}[n, m]$ nanotube Figure 1 is given by

$$
\begin{aligned}
F\left(\overline{\mathrm{TiO}_{2}[n, m]}\right)= & (6 m n+6 n)(6 m n+6 n-1)^{3}-4(10 m n+8 n)(6 m n+6 n-1)^{2} \\
& +3(6 m n+6 n-1)(76 m n+48 n)-320 m n-160 n .
\end{aligned}
$$

Proof. By Proposition 1, we have $F(\bar{G})=n(n-1)^{3}-4 m(n-1)^{2}+3(n-1) M_{1}(G)-F(G)$, and since $F\left(\mathrm{TiO}_{2}[n, m]\right)=320 m n+160 n$, and $\mathrm{M}_{1}\left(\mathrm{TiO}_{2}[n, m]\right)=76 m n+48 n$ given in [16]. In [17] the partitions of the vertex set and edge set $\sum\left|V\left(\mathrm{TiO}_{2}[n, m]\right)\right|=6 m n+6 n, \sum\left|E\left(\mathrm{TiO}_{2}[n, m]\right)\right|=10 m n+8 n$ of titania nanotubes. Then

$$
\begin{aligned}
F\left(\overline{\mathrm{TiO}_{2}[n, m]}\right)= & \sum\left|V\left(\mathrm{TiO}_{2}[n, m]\right)\right|\left(\sum\left|V\left(\mathrm{TiO}_{2}[n, m]\right)\right|-1\right)^{3}-4 \sum\left|E\left(\mathrm{TiO}_{2}[n, m]\right)\right|\left(\sum|V(\mathrm{TiO} 2[n, m])|-1\right)^{2} \\
& +3\left(\sum\left|V\left(\mathrm{TiO}_{2}[n, m]\right)\right|-1\right) \mathrm{M}_{1}\left(\mathrm{TiO}_{2}[n, m]\right)-F\left(\mathrm{TiO}_{2}[n, m]\right) \\
= & (6 m n+6 n)(6 m n+6 n-1)^{3}-4(10 m n+8 n)(6 m n+6 n-1)^{2} \\
& +3(6 m n+6 n-1)(76 m n+48 n)-320 m n-160 n
\end{aligned}
$$




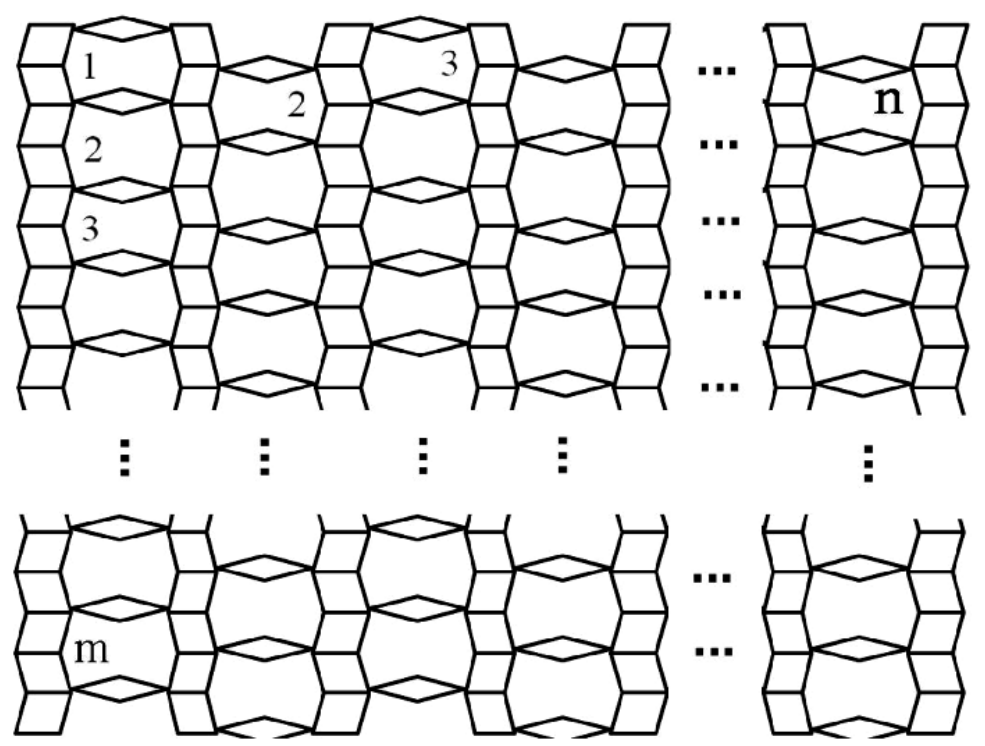

Figure 1. 2-Dimensional Lattice of molecular graph of $\mathrm{TiO}_{2}[n, m]$ nanotube $(n, m \in \mathbb{N})$.

Corollary 2. Let $T=T[p, q]$ be the molecular graph of a nanotorus such that $|V(T)|=p q,|E(T)|=\frac{3}{2} p q$, Figure 2 . Then
a. $F(\overline{T[p, q]})=p q\left[(p q-1)^{2}(p q-7)+27(p q-1)-27\right]$.
b. $F\left(P_{n} \times T\right)=p q[125 n-122]$.
c. $F\left(\overline{P_{n} \times T}\right)=p q\left[(n p q-1)^{2}\left(n^{2} p q-11 n+4\right)+3(n p q-1)(25 n-18)-125 n+122\right]$.

Proof. a. By Proposition 1, we have $F(\bar{G})=n(n-1)^{3}-4 m(n-1)^{2}+3(n-1) M_{1}(G)-F(G)$, and since $M_{1}(T)=9 p q$ given in [14]. and $F(T)=27 p q$ given in [18]. Then

$$
\begin{aligned}
F(\overline{T[p, q]}) & =|V(T)|(|V(T)|-1)^{3}-4|E(T)|(|V(T)|-1)^{2}+3(|V(T)|-1) M_{1}(T)-F(T) \\
& =p q(p q-1)^{3}-6 p q(p q-1)^{2}+27 p q(p q-1)-27 p q \\
& =p q\left[(p q-1)^{2}(p q-7)+27(p q-1)-27\right] .
\end{aligned}
$$

b. By $F\left(G_{1} \times G_{2}\right)=n_{2} F\left(G_{1}\right)+n_{1} F\left(G_{2}\right)+6 m_{2} M_{1}\left(G_{1}\right)+6 m_{1} M_{1}\left(G_{2}\right)$, given in [6]. and since $M_{1}\left(P_{n}\right)=$ $4 n-6, M_{1}(T)=9 p q$ given in [14]. and $F\left(P_{n}\right)=8 n-14, F(T)=27 p q$ given in [18]. Then

$$
\begin{aligned}
F\left(P_{n} \times T\right) & =|V(T)| F\left(P_{n}\right)+\left|V\left(P_{n}\right)\right| F(T)+6|E(T)| M_{1}\left(P_{n}\right)+6\left|E\left(P_{n}\right)\right| M_{1}(T) \\
& =2 p q(4 n-7)+27 n p q+18 p q(2 n-3)+54 p q(n-1) \\
& =p q[125 n-122] .
\end{aligned}
$$

c. By [19], $M_{1}\left(P_{n} \times T\right)=p q(25 n-18)$, and by using Lemma 1, $\left|E\left(P_{n} \times T\right)\right|=p q\left(\frac{5}{2} n-1\right),\left|V\left(P_{n} \times T\right)\right|=$ $n p q$, and applying Proposition 1 and item (b) we get

$$
\begin{aligned}
F\left(\overline{\left.P_{n} \times T\right)}=\right. & \left|V\left(P_{n} \times T\right)\right|\left(\left|V\left(P_{n} \times T\right)\right|-1\right)^{3}-4\left|E\left(P_{n} \times T\right)\right|\left(\left|V\left(P_{n} \times T\right)\right|-1\right)^{2} \\
& +3\left(\left|V\left(P_{n} \times T\right)\right|-1\right) M_{1}\left(P_{n} \times T\right)-F\left(P_{n} \times T\right) \\
= & n p q(n p q-1)^{3}-4 p q\left(\frac{5}{2} n-1\right)(n p q-1)^{2} \\
& +3 p q(n p q-1)(25 n-18)-p q[125 n-122] \\
= & p q\left[(n p q-1)^{2}\left(n^{2} p q-11 n+4\right)+3(n p q-1)(25 n-18)-125 n+122\right] .
\end{aligned}
$$




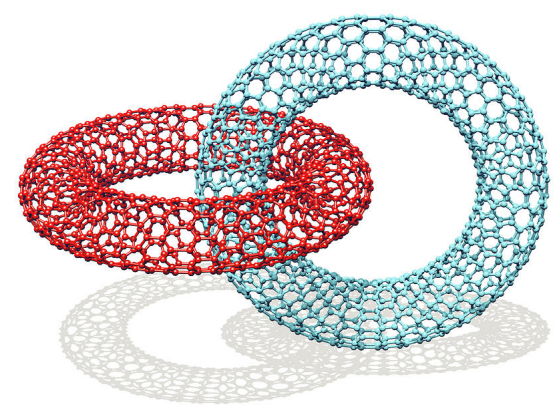

Figure 2. Molecular graph of a nanotorus

\section{Conclusion}

The forgotten index one of the most important topological indices which preserve the symmetry of molecular structures and provide a mathematical formulation to predict their physical and chemical properties. In this article, we computed the forgotten index of some basic mathematical operations and obtained explicit formula for their values under complement graph operations, and we computed the forgotten index of molecular complement graph of nanotorus and titania nanotubes $\mathrm{TiO}_{2}[n, m]$.

Author Contributions: All authors contributed equally to the writing of this paper. All authors read and approved the final manuscript.

Conflicts of Interest: "The authors declare no conflict of interest."

\section{References}

[1] Alsharafi, M., Shubatah, M., \& Alameri, A. (2020). The hyper-Zagreb index of some complement graphs. Advances in Mathematics: Scientific Journal, 9(6), 3631-3642.

[2] Gutman, I., \& Trinajstić, N. (1972). Graph theory and molecular orbitals. Total $\pi$-electron energy of alternant hydrocarbons. Chemical Physics Letters, 17(4), 535-538.

[3] Ashrafi, A. R., Došlić, T., \& Hamzeh, A. (2010). The Zagreb coindices of graph operations. Discrete Applied Mathematics, 158(15), 1571-1578.

[4] Furtula, B., \& Gutman, I. (2015). A forgotten topological index. Journal of Mathematical Chemistry, 53(4), $1184-1190$.

[5] De, N., Nayeem, S. M. A., \& Pal, A. (2016). The F-coindex of some graph operations. SpringerPlus, 5(1), 221.

[6] De, N., Nayeem, S. M. A., \& Pal, A. (2016). F-index of some graph operations. Discrete Mathematics, Algorithms and Applications, 8(02), 1650025.

[7] Alameria, A., Al-Naggara, N., Al-Rumaima, M., \& Alsharafi, M. (2020). Y-index of some graph operations. International Journal of Applied Engineering Research (IJAER), 15(2), 173-179.

[8] Alameri, A., Shubatah, M., \& Alsharafi, M. (2020). Zagreb indices, Hyper Zagreb indices and Redefined Zagreb indices of conical graph. Advances in Mathematics: Scientific Journal, 9(6), 3631-3642.

[9] Ayache, A., Ghallab, A. A. A., \& Modabish, A. (2020). Wiener polynomial and Wiener index of conical graphs. Sylwan, 164(3). 107-116.

[10] Behmaram, A., Yousefi-Azari, H. \& Ashrafi, A. (2011). Some New Results on Distance-Based Polynomials, MATCH Communications in Mathematical and in Computer Chemistry, 65, 39-50.

[11] Alsharafi, M., Shubatah, M. \& Alameri, A. (2020). The First and Second Zagreb Index of Complement Graph and Its Applications of Molecular Graph, Asian Journal of Probability and Statistics, 8(3), 15-30.

[12] Gutman, I. (2017). On hyper-Zagreb index and coindex. Bulletin (Académie serbe des sciences et des arts. Classe des Sciences Mathématiques et Naturelles. Sciences mathématiques), (42), 1-8.

[13] Kiruthika, K. (2016). Zagreb indices and Zagreb coindices of some graph operations. International Journal of Advanced Research in Engineering and Technology, 7(3), 25-41.

[14] Khalifeh, M. H., Yousefi-Azari, H., \& Ashrafi, A. R. (2009). The first and second Zagreb indices of some graph operations. Discrete Applied Mathematics, 157(4), 804-811.

[15] Alsharafi, M. S., Shubatah, M. M., \& Alameri, A. Q. (2020). On the Hyper-Zagreb coindex of some graphs. Journal of Mathematics and Computer Science, 10(5), 1875-1890.

[16] De, N. (2016). On Molecular Topological Properties of $\mathrm{TiO}_{2}$ Nanotubes, Journal of Nanoscience, Article ID 1028031. 
[17] Malik, M. A. \& Imran, M. (2015). On Multiple Zagreb Indices of $\mathrm{TiO}_{2}$ Nanotubes, Acta Chimica Slovenica, $62,973-976$.

[18] Aram, H. \& Dehgardi, N. (2017). Reformulated F-index of graph operations, Communications in Combinatorics and Optimizatio 1(2), 87-98.

[19] Alsharafi, M. \& Shubatah, M. (2020). On the Hyper-Zagreb index of some Graph Binary Operations, Asian Research Journal of Mathematics 16(4), 12-24.

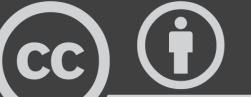

BY

(C) 2020 by the authors; licensee PSRP, Lahore, Pakistan. This article is an open access article distributed under the terms and conditions of the Creative Commons Attribution (CC-BY) license (http://creativecommons.org/licenses/by/4.0/). 